\title{
Persönliches
}

\section{Wir gratulieren}

\section{zum 30. Geburtstag}

Herrn Dipl.-Ing. Florian Alber Herrn Dipl.-Ing. Andreas Frank Herrn Ing. Ronald Hanke Herrn Alexander Hatzel Herrn Dipl.-Ing. Eugen Konzett Herrn Thomas Lücker Herrn Dipl.-Ing. Walter Mayrhofer Herrn Ing. Josef Maxones Herrn Dipl.-Ing. Michael Zimonic

\section{zum 35. Geburtstag}

Herrn Ing. Oswald Fuchssteiner Herrn Dipl.-Ing. Rudolf Herndlhofer Herrn Dipl.-Ing. Christian Hölzl Herrn Dipl.-Ing. Amandus Kofler Herrn Dipl.-Ing. Dr. Andreas Magauer

\section{zum 40. Geburtstag}

Herrn Dipl.-Ing. Dr. Gerhard Diendorfer Herrn Johann Gruber

Herrn Anton Gschaider

Herrn Dipl.-Ing. Anton Haumer

Herrn Univ.-Doz. Dipl.-Ing.

Dr. Franz Hofbauer

Herrn Dipl.-Ing. Alfred Leeb

Herrn Ing. Robert Ludwig

Herrn Dipl.-Ing. Martin Piehslinger

\section{zum 45. Geburtstag}

Herrn Ing. $\mathrm{H}$. Ischovitsch Dolischo Herrn Dipl.-Ing. Branko Nenadovic Herrn Ing. Robert Neumann

\section{zum 50. Geburtstag}

Herrn Dipl.-Ing. Dr. Günter Moraw

\section{zum 55. Geburtstag}

Herrn Prok. Alfred Horvath

Herrn Dipl.-Ing. Werner Katschinka

Herrn Prof. Ziv.-Ing. f. ET

Dipl.-Ing. Gerhard Pranger

zum 60. Geburtstag

Herrn Ziv.-Ing. f. ET

Dipl.-Ing. Friedrich Turnovszky

\section{zum 65. Geburtstag}

Herrn Dir. i. R. Dipl.-Ing. Johann Lehner Herrn Prof. Ziv.-Ing. f. ET

Dipl.-Ing. Hardo Stadler

und wünschen alles Gute!

\section{Buchbesprechungen}

\begin{abstract}
Volkmann, P.: Taschenbuch Elektrotechnik + Elektronik. Formeln - Tabellen - Erläuterungen. Band 2: Fachkenntnisse. 2. überarbeitete und erweiterte Auflage. 372 S. VDE, Berlin, 1996. Kart. DM 59,-, sFr 59,-, S 437,--.

Das vorliegende Buch ist eine Formelund Tabellensammlung mit Erläuterungen. Es werden dabei die Gebiete der allgemeinen Elektrotechnik, beginnend vom Wechsel- und Drehstrom, elektrische Meßtechnik, Transformatoren, Maschinen, Lichttechnik, Bemessung von Leitungen bis zu den elektrischen Schutzmaßnahmen besprochen. Auch die Grundlagen der Elektronik und Digitaltechnik werden behandelt. Die angeführten Formeln und Tabellen sind sehr umfassend und übersichtlich angeordnet. Das Auffinden wird durch eine klare Gliederung und ein eigenes Tabellenund Sachwortverzeichnis unterstützt.
\end{abstract}

Nachdem es sich um eine deutsche Publikation handelt, ist bei der Bezugnahme auf Gesetze und Bestimmungen im Einzelfall zu prüfen, ob diese auch für Österreich zutreffend sind.
Das Buch stellt ein sehr wertvolles Nachschlagwerk für Auszubildende, aber auch für Praktiker und sonst elektrotechnisch Interessierte dar.

\section{Robert Seufert ÖVE}

Marinescu, M.: Elektrische und magnetische Felder. Eine praxisorientierte Einführung. $260 \mathrm{Abb}$., XIII, $286 \mathrm{~S}$. Springer, Berlin Heidelberg New York London Paris Tokyo Hong Kong, 1996. Brosch. DM 39,-, S 284,70, sFr 39,--

Wie aus dem Untertitel hervorgeht, wird mit möglichst geringem mathematischem Aufwand versucht, die Grundtatsachen des elektrischen und magnetischen Feldes zu vermitteln. Der Leser muß allerdings sowohl mit Vektoralgebra als auch mit Differential- und Integralrechnung vertraut sein.

In den ersten beiden Kapiteln werden elektrostatische und stationäre elektrische Felder behandelt. Ihr Fachwissen als Magnetikerin hat die Autorin in das umfangreich behandelte Kapitel über stationäre magnetische Felder eingear- beitet. Besonders erwähnenswert sind daran die mit der Methode der finiten Elemente berechneten sehr anschaulichen Feldbilder. Im letzten Kapitel werden die zeitlich langsam veränderlichen Felder besprochen. Zur Vertiefung des Stoffs sind 35 ausführlich durchgerechnete Beispiele dem Text beigefügt.

Das Buch, das nach Vorlesungen der Verfasserin an der Fachhochschule Wiesbaden entstand, kann speziell Studenten an Fachhochschulen und in der Praxis stehenden Ingenieuren empfohlen werden.

Herbert Haas

Bonfig, K.W.: Temperatur-Sensoren. Prinzipien und Applikationen. (Reihe Sensorik, Bd. 6.) 135 Abb., 21 Tab., 187 S. expert, Renninen, 1995. DM 78,-

Die Temperatur ist eine umfassend wichtige Meßgröße, ebenso als eigentliche Meßgröße wie als alles beeinflussender Umgebungsparameter. Es sind Werte aus einem extrem weiten Bereich zu mes- 